\title{
TITLE:
}

\section{Lower-Stratospheric Radiative Damping and Polar-Night Jet Oscillation Events}

\section{$\operatorname{AUTHOR}(\mathrm{S})$ :}

Hitchcock, Peter; Shepherd, Theodore G.; Taguchi, Masakazu; Yoden, Shigeo; Noguchi, Shunsuke

\section{CITATION:}

Hitchcock, Peter ...[et al]. Lower-Stratospheric Radiative Damping and Polar-Night Jet Oscillation Events. Journal of the Atmospheric Sciences 2013, 70(5): 1391-1408

ISSUE DATE:

2013-05

URL:

http://hdl.handle.net/2433/218335

RIGHT:

(C) 2013 American Meteorological Society 


\title{
Lower-Stratospheric Radiative Damping and Polar-Night Jet Oscillation Events
}

\author{
Peter Hitchcock And TheOdore G. ShePherd \\ Department of Physics, University of Toronto, Toronto, Ontario, Canada \\ MASAKAZU TAGUCHI \\ Department of Earth Sciences, Aichi University of Education, Kariya, Japan \\ Shigeo Yoden AND SHunsuke Noguchi \\ Division of Earth and Planetary Sciences, Graduate School of Science, Kyoto University, Kyoto, Japan
}

(Manuscript received 5 July 2012, in final form 14 November 2012)

\begin{abstract}
The effect of stratospheric radiative damping time scales on stratospheric variability and on stratospheretroposphere coupling is investigated in a simplified global circulation model by modifying the vertical profile of radiative damping in the stratosphere while holding it fixed in the troposphere. Perpetual-January conditions are imposed, with sinusoidal topography of zonal wavenumber 1 or 2.

The depth and duration of the simulated sudden stratospheric warmings closely track the lower-stratospheric radiative time scales. Simulations with the most realistic profiles of radiative damping exhibit extended timescale recoveries analogous to polar-night jet oscillation (PJO) events, which are observed to follow sufficiently deep stratospheric warmings. These events are characterized by weak lower-stratospheric winds and enhanced stability near the tropopause, which persist for up to 3 months following the initial warming. They are obtained with both wave- 1 and wave- 2 topography. Planetary-scale Eliassen-Palm (EP) fluxes entering the vortex are also suppressed, which is in agreement with observed PJO events.

Consistent with previous studies, the tropospheric jets shift equatorward in response to the warmings. The duration of the shift is closely correlated with the period of enhanced stability. The magnitude of the shift in these runs, however, is sensitive only to the zonal wavenumber of the topography. Although the shift is sustained primarily by synoptic-scale eddies, the net effect of the topographic form drag and the planetary-scale fluxes is not negligible; they damp the surface wind response but enhance the vertical shear. The tropospheric response may also reduce the generation of planetary waves, further extending the stratospheric dynamical time scales.
\end{abstract}

\section{Introduction}

The lower polar stratosphere has been identified as a key region for the two-way coupling between the stratosphere and the troposphere. Circulation anomalies in the stratospheric polar vortices in both hemispheres have been shown to influence the extratropical tropospheric jets, whether they are caused by, for instance, ozone depletion in the Southern Hemisphere (Thompson and Solomon 2002; Son et al. 2008) or

Corresponding author address: Peter Hitchcock, Department of Applied Mathematics and Theoretical Physics, Centre for Mathematical Sciences, Wilberforce Road, Cambridge CB3 0WA, United Kingdom.

E-mail:aph42@cam.ac.uk sudden warmings in the Northern Hemisphere (Baldwin and Dunkerton 2001).

This coupling is of interest for several reasons. First, it provides a potential pathway for a variety of stratospheric forcings to influence the surface climate. Second, the stratospheric circulation has been shown to have significantly longer decorrelation time scales than does the troposphere, suggesting the former may be a source of additional skill for seasonal prediction of the latter (Baldwin et al. 2003). Forecasting studies, however, suggest that in the Northern Hemisphere this enhanced predictability arises only following certain sudden warmings and is not uniform in time (Mukougawa et al. 2009).

One source of the seasonal-scale predictability in the stratosphere is likely to be the slow, downward migration 
of zonal temperature and wind anomalies, which have been termed the polar-night jet oscillation (PJO) by Kuroda and Kodera (2001). This slow variability has recently been shown to be quite a robust behavior of the vortex following particularly deep warmings (Hitchcock et al. 2013), suggesting that PJO events are best thought of as an extended recovery from such major warmings. The duration of these recoveries is strongly correlated with the depth to which the circulation is disturbedthose events that disturb the lowermost stratosphere persist the longest. This corresponds to the vertical gradient in radiative damping time scales, which reach their longest in the lowermost stratosphere as well (Dickinson 1973). The recoveries, moreover, are also characterized by the strong suppression of planetary wave propagation into the vortex. Since radiatively driven processes are likely to be much more predictable than the strongly nonlinear wave driving, this suggests at least a proximate reason for the enhanced predictability.

The present study, then, is in part motivated by an attempt to better understand the dynamics of these PJO events in the context of a simplified, "mechanistic" circulation model, of the type motivated by Held and Suarez (1994). Such models have been an essential tool in characterizing the range of possible behavior of the fully coupled stratosphere-troposphere system (Taguchi et al. 2001; Yoden et al. 2002), since their simple parameterizations result in relatively fast execution times, permitting key parameters to be swept through plausible ranges. The possible influence of the time-mean strength of the stratospheric vortex on the position of the tropospheric jet was highlighted dramatically by Polvani and Kushner (2002). While the sensitivity was subsequently found to be unrealistically large owing to the unrealistically long decorrelation time scales in their tropospheric jet, Gerber and Polvani (2009) nonetheless confirmed the poleward shift of the tropospheric jet in response to the radiative strengthening of the stratospheric vortex.

Radiative heating is commonly parameterized in this class of models as a simple Newtonian relaxation to a prescribed radiative equilibrium temperature (Held and Suarez 1994; Yoden et al. 2002). The role of lowerstratospheric radiative damping is, therefore, a natural candidate for a parameter sweep experiment. Indeed, a variety of vertical profiles of damping time scales have been employed, and several sensitivity studies have been carried out. Two profiles in particular are commonly employed. A vertically constant time scale was employed by Polvani and Kushner (2002), Gerber and Polvani (2009), and Scott and Polvani (2006), with values ranging from 5 to 40 days. The vertical profile of time scales adopted by Holton and Mass (1976) falls from 25 days in the lower stratosphere to 5 days in the upper stratosphere, and was employed by Taguchi et al. (2001) and Scott and Polvani (2006). The latter found that power in the spectrum of variability produced in a perpetual-January run generally shifted toward lower frequencies as the radiative time scales lengthened, though the behavior was a complicated function of the height of the imposed surface topography. In a study closely related to the present work, the sensitivity of tropospheric annular mode decorrelation time scales to four separate profiles of stratospheric damping times was investigated by Charlton-Perez and O'Neill (2010, hereafter $\mathrm{CO}$ ), revealing relatively little sensitivity of the tropospheric dynamics to the stratospheric radiative time scales. Given the arguments above regarding the relevance of the radiative time scales to stratospheric dynamical time scales and the influence of the latter on tropospheric time scales seen in a comprehensive model (Simpson et al. 2011), this result is unexpected.

In a recent study, Hitchcock et al. (2010) demonstrated that such a Newtonian relaxation scheme can realistically describe heating rates in the middle atmosphere, sufficiently so that effective time scales can be diagnosed from a comprehensive chemistry-climate model. The analysis demonstrated that the relevant radiative time scales can reach 70-80 days in the lower Arctic stratosphere, which is considerably longer than the values typically used in mechanistic circulation studies. Since this comprehensive model-based estimate is likely to lie closer to the true radiative time scales [see Hitchcock et al. (2010) for a comparison with other estimates], this suggests that it is worth reexamining the effect of radiative time scales on stratospheric-vortex variability, and provides an additional motivation for the present study.

In contrast to $\mathrm{CO}$, the parameter sweep considered here produces a broad spectrum of sudden warming event durations, with the longest and most realistic profiles inducing variability quite analogous to observed PJO events. This clearly demonstrates the relevance of the radiative time scales to the variability. Moreover, this spectrum of variability provides a useful context for investigating the tropospheric impact of sudden warmings. While the warmings themselves are induced by the coupling between the stratosphere and troposphere, in this context they can be thought of as a "forcing" external to the tropospheric jets themselves, whose dynamics are driven primarily by synoptic-scale eddies confined to the troposphere.

The dynamics of the tropospheric jets in response to various external forcings is a rich and active area of study [see Kushner (2010) for a recent review], and a detailed consideration of the dynamics of the tropospheric response is beyond the scope of the present study. The 
intent here is simply to document the magnitude and duration of the tropospheric response to the spectrum of warmings, and to point out the perhaps underappreciated role that the rearrangement of tropospheric planetary-scale momentum fluxes and form drag generated by the topography can play in this response.

Details of the model configuration, including the vertical profiles of radiative damping time scales, are provided in the second section. A complication arising from the present methodology is that when the radiative damping time scales are changed, not only is the variability changed, but also the time-mean circulation. An important task in the present analysis is therefore to distinguish between these two effects. To this end, the time-mean response of the circulation is described in the third section. A characterization of the stratospheric variability and the associated tropospheric response follows in the fourth section. Conclusions and discussion are presented in the final section.

\section{Model setup}

The model employed here is the same as that of Taguchi et al. (2001), to which the reader is referred for complete details. The dynamical core is run at a horizontal resolution of $\mathrm{T} 21$, with 42 vertical levels from the surface through the mesosphere. Radiation is

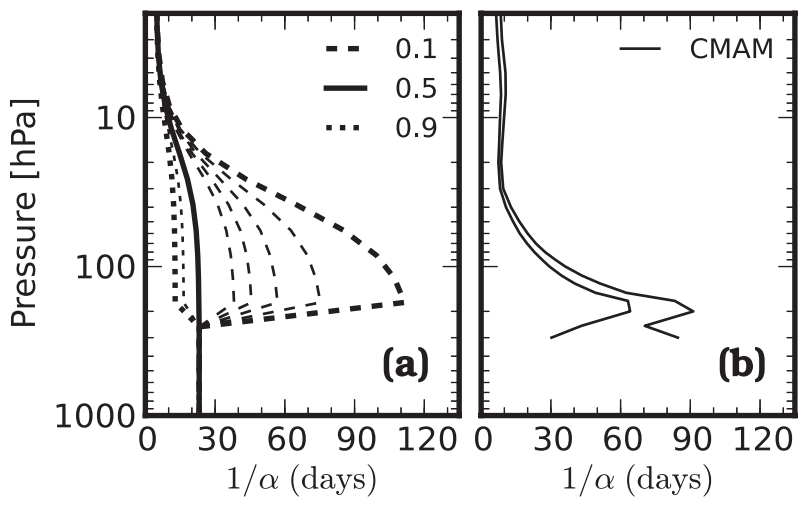

FIG. 1. (a) Vertical profiles of radiative damping time scales (days). The control profile is shown as a solid thick line. The thin dotted line corresponds to $\alpha_{\mathrm{LS}}=0.7$, while the thin dashed lines correspond to $\alpha_{\mathrm{LS}}=0.3,0.25,0.2$, and 0.15 , from left to right. (b) Profile of middle-atmosphere radiative time scales in the Arctic winter estimated from CMAM (see text). The two lines show the $95 \%$ confidence interval.

parameterized by Newtonian relaxation toward the same equilibrium temperature field appropriate for a persistent-January configuration used by Taguchi et al. (2001). The control runs make use of the same vertical profile of radiative damping rates, adopted from that used by Holton and Mass (1976). We perturb the vertical profile according to the following:

$$
\alpha= \begin{cases}\left(\alpha_{\mathrm{LS}}+\frac{1}{2}\left(\alpha_{\mathrm{US}}-\alpha_{\mathrm{LS}}\right)\left[1+\tanh \left(\frac{z-35 \mathrm{~km}}{7 \mathrm{~km}}\right)\right]\right) \times 10^{-6} \mathrm{~s}^{-1} & \text { if } z>10 \mathrm{~km} \\ \alpha_{T} \times 10^{-6} \mathrm{~s}^{-1} & \text { otherwise }\end{cases}
$$

The lower-stratospheric damping rates are controlled by $\alpha_{\mathrm{LS}}$; simulations are performed at the following values: $0.1,0.15,0.2,0.25,0.3,0.5,0.7$, and 0.9 ; these profiles are shown in Fig. 1. For comparison, also shown is an estimate of the damping rates based on simulations of the Canadian Middle Atmosphere Model (CMAM), a comprehensive chemistry-climate model (Hitchcock et al. 2010). The value of $\alpha_{\mathrm{LS}}=0.5$ corresponds to the profile used by Taguchi et al. (2001); this simulation is therefore considered the control run. The layer in the lower stratosphere with long damping time scales is somewhat deeper in the idealized profiles than in CMAM, but it is clear that the lower-stratospheric damping time scales considered here are well justified by the diagnosed values. For comparison, the profiles considered by $\mathrm{CO}$ had a maximum lower-stratospheric time scale of 40 days. The emphasis in the present analysis will be on the weakened damping runs. Furthermore, we contrast in detail the weakest damping run $\left(\alpha_{\mathrm{LS}}=0.1\right)$ with the control run, though we note that the value of $\alpha_{\mathrm{LS}}=0.15$ best matches the effective damping exhibited by CMAM. The details are not strongly sensitive to this choice.

The parameters determining the upper-stratospheric damping and the tropospheric damping are held at values used by Taguchi et al. (2001): $\alpha_{\mathrm{US}}=2.5$ and $\alpha_{T}=0.5$, respectively. This results in tropospheric damping time scales of approximately 25 days. Note that this is stronger than the 40-day damping time scale specified in Held and Suarez (1994).

Two sets of experiments have been performed, with sinusoidal surface topography of zonal wavenumbers 1 and 2 specified in the Northern Hemisphere. The meridional profile in all cases is quartic in $\sin \phi$ with a maximum of $1000 \mathrm{~m}$ at $45^{\circ} \mathrm{N}$; the analytical form is the same as that of Taguchi et al. (2001). Surface friction is imposed as a linear damping on the lowest model level with a rate of $0.5 \mathrm{day}^{-1}$. Rayleigh drag is also imposed above $50 \mathrm{~km}$ as a sponge layer. The model simulations are run for 

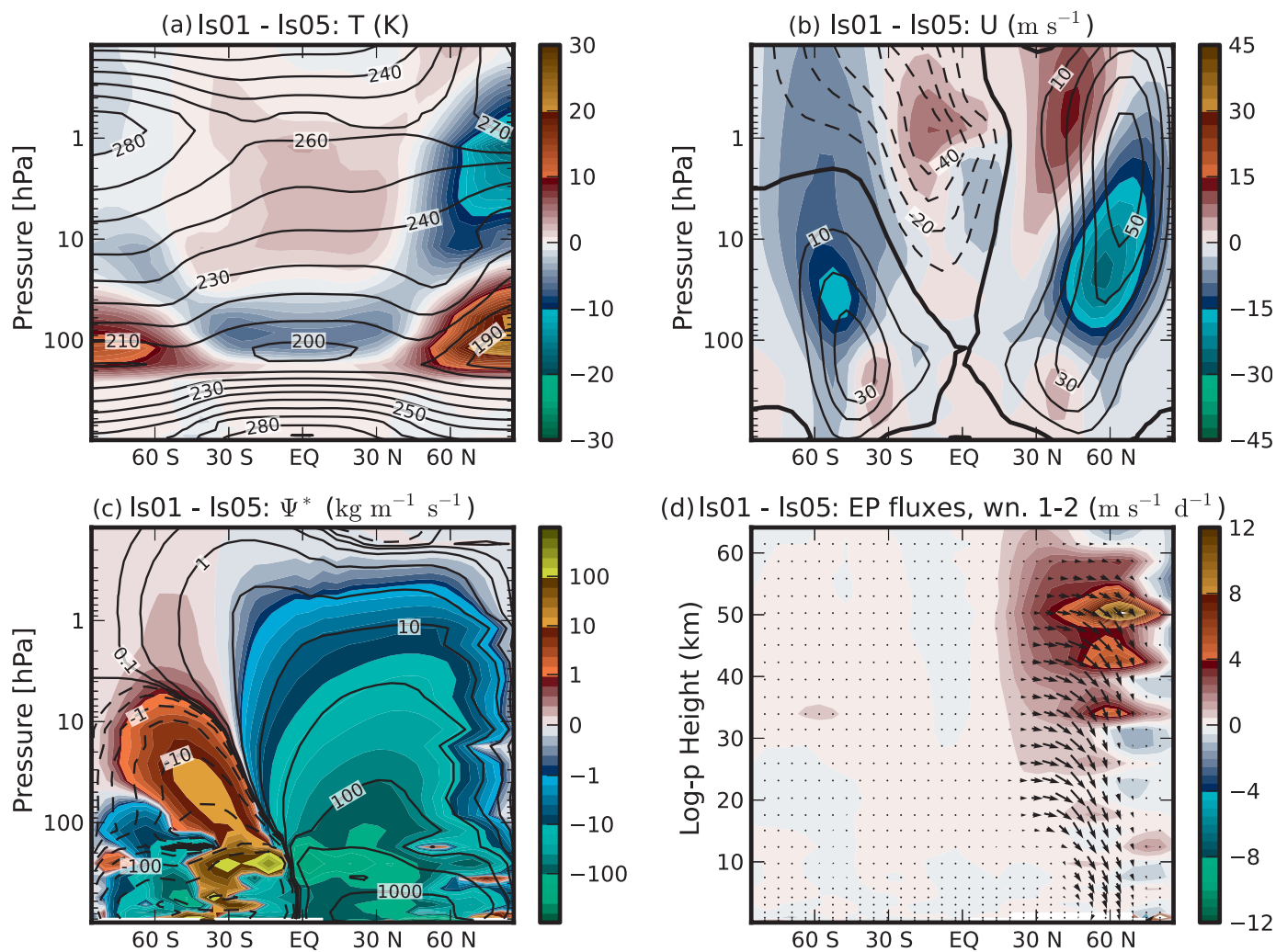

FIG. 2. (a) Climatological zonal-mean temperatures from the wave-1 control simulation (contour lines) and the difference in the weakened damping run (filled contours). Contour lines are at an interval of $10 \mathrm{~K}$. (b) As in (a), but for zonal-mean zonal winds. Contour lines are at intervals of $10 \mathrm{~m} \mathrm{~s}^{-1}$. (c) As in (a), but for the TEM streamfunction. Contour shading and lines are logarithmically spaced between 0.1 and $1000 \mathrm{~kg} \mathrm{~m}^{-1} \mathrm{~s}^{-1}$. (d) Difference in EP fluxes (arrows) and their divergence (filled contours) between the weakened damping run and the control run.

10200 days with the first 200 days discarded for spinup. Two additional simulations at the weakest damping rate $\alpha_{\mathrm{LS}}=0.1$ are run for 100200 days to improve composite statistics as discussed further below. Except where noted, the 10 200-day runs are used for consistency.

Although some concerns have been raised regarding simulations performed at this relatively low horizontal resolution (Gerber et al. 2008), the use of topography in the present runs results in tropospheric northern annular mode (NAM) time scales on the order of 20 days, comparable to the real atmosphere. In contrast, the time scales of the southern annular mode (SAM) in the absence of topography are found to be on the order of 40 days in these simulations. These time scales are discussed further in section $4 \mathrm{~d}$.

\section{Time-mean response}

\section{a. Stratospheric changes}

The time-mean changes induced by weakening the radiative damping are summarized for the wave- 1 topography runs in Fig. 2 and for the wave-2 topography runs in Fig. 3. Henceforth these will be referred to as wave- 1 and wave- 2 runs, respectively. Relevant dynamical quantities are computed in the transformed Eulerian mean (TEM) framework following Andrews et al. (1987). The coarse details are quite similar for the two sets of experiments. The direct impact (and the dominant one, in these experiments) of weakening the radiative damping in the lower stratosphere is to warm the poles, which are subject to adiabatic heating, and to cool the tropics, which are subject to adiabatic cooling. Figures $2 \mathrm{a}$ and $3 \mathrm{a}$ show the control run climatological temperatures (contour lines), and the change induced by weakening the damping rates (shaded contours). The polar regions in the lower stratosphere warm, and the tropics cool, as expected. In balance with this weakened equator-to-pole temperature gradient, the polar-night jet weakens (Figs. 2b and 3b). In addition to this direct response, however, the planetary-scale eddies also adjust, providing a negative dynamical feedback. The residual (TEM) circulation weakens (Figs. 2c and 3c) as a result of the weakened planetary-scale wave driving 
(a) Is01m2 - Is05m2: T (K)

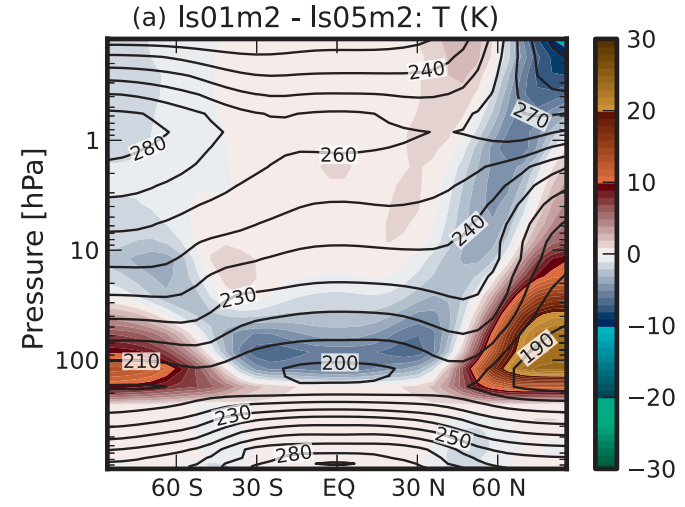

(c) Is01m2 - Is05m2: $\Psi^{*}\left(\mathrm{~kg} \mathrm{~m}^{-1} \mathrm{~s}^{-1}\right)$

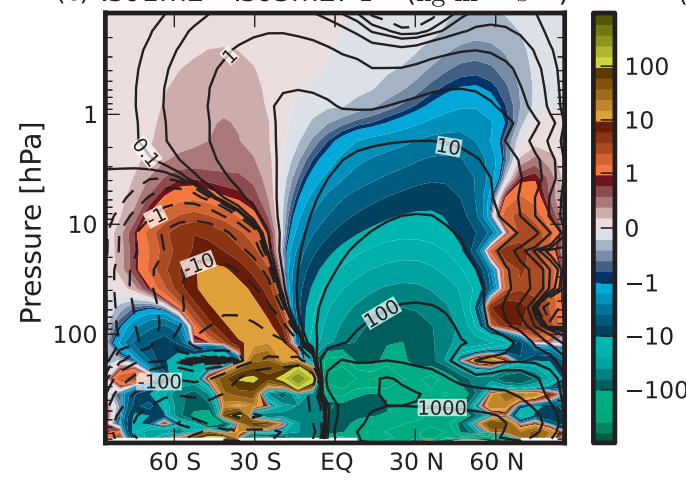

(b) Is01m2 - Is05m2: U $\left(\mathrm{m} \mathrm{s}^{-1}\right)$

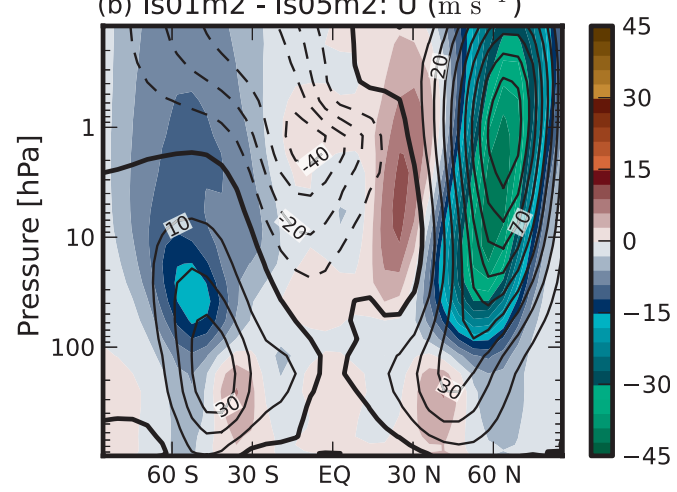

(d) Is01m2 - Is05m2: EP fluxes, wn. 1-2 (m s $\left.{ }^{-1} \mathrm{~d}^{-1}\right)$

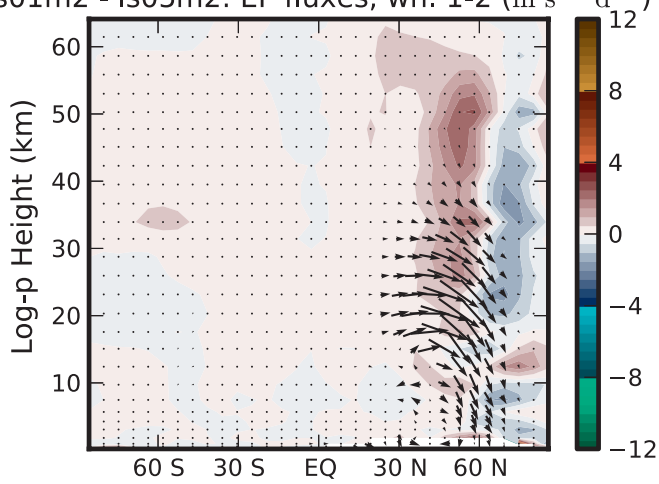

FIG. 3. As in Fig. 2, but for the wave-2 topography simulations.

(Figs. 2d and 3d). This response is most likely dominated by the response of the waves to the new zonal-mean state. While the waves will also be directly affected by the weakened damping rates, were this the dominant effect they would be expected to propagate higher into the stratosphere. This feedback induces changes higher in the stratosphere, warming the tropical upper stratosphere where the upwelling decreases, and cooling the high-latitude upper stratosphere where the downwelling decreases. The cooling is present in both hemispheres but is much stronger in the Northern Hemisphere.

The climatological stratospheric jet in the wave- 2 runs is considerably stronger than that in the wave- 1 runs, and the changes in the winds induced by the weakened damping rates are more barotropic. This can be understood as a consequence of the greater tendency of the shorter wavelength waves to be refracted equatorward (Karoly and Hoskins 1982). The climatological planetaryscale eddy fluxes in the wave-2 control run do not deposit their momentum in altitudes or latitudes as high as those in the wave- 1 run. While the overall fluxes are weaker in the wave- 2 weakened damping run, more flux reaches high latitudes, leading to greater flux convergence over the pole (Fig. 3d), which shifts the downwelling branch of the meridional circulation poleward (Fig. 3c).
The tropospheric jets in both series of experiments also shift equatorward in both hemispheres. This is consistent with the response to transient stratospheric warming in the real atmosphere (Baldwin and Dunkerton 2001) and with the response found to time-averaged diabatic forcings in other studies (Polvani and Kushner 2002; Haigh et al. 2005; Gerber and Polvani 2009). This shift is considered in greater detail below; here we note simply that the shift in the Southern Hemisphere jet is stronger than that in the Northern Hemisphere jet in the wave- 1 simulations, despite the weaker temperature changes, and that the shift in the jets in the wave- 2 simulations is stronger than that in the wave- 1 simulations.

To consider these changes somewhat more quantitatively, Fig. 4 shows several summary statistics for these runs as a function of $\alpha_{\mathrm{LS}}$. Confidence intervals at the $95 \%$ significance level are estimated by a modified Student's $t$ test, in which the degrees of freedom are modified to take serial correlations into account. Details of this test can be found in the appendix.

Under quasigeostrophic scaling assumptions, the temperature anomaly in steady state is determined by the balance between radiative cooling and adiabatic heating. The direct effect of weakening the damping rates 
(a)

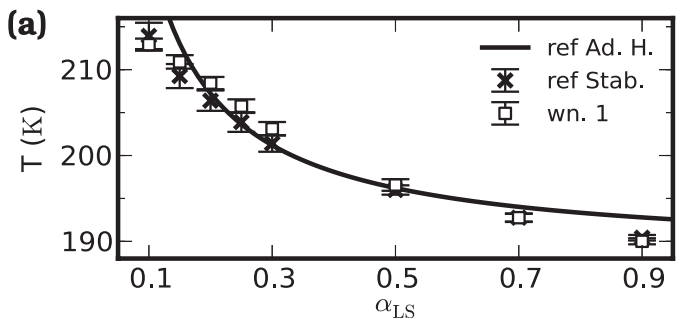

(c)

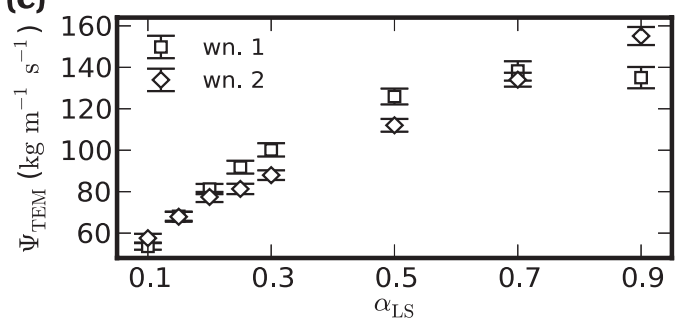

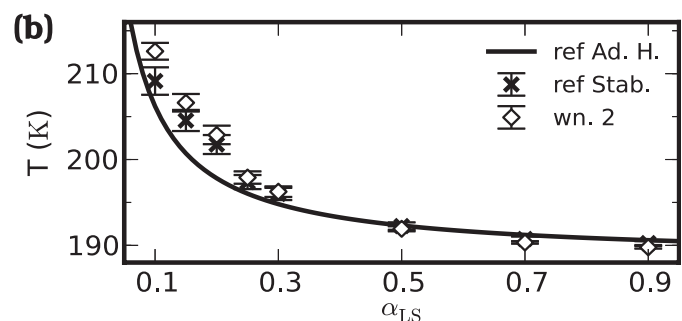

(d)

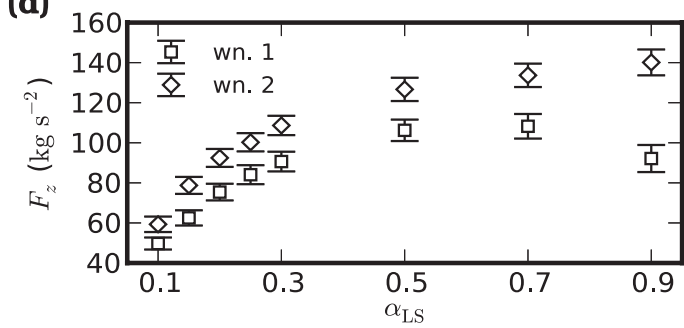

FIG. 4. Polar cap-averaged $\left(60^{\circ}-90^{\circ} \mathrm{N}\right)$ temperatures for (a) wave- 1 and (b) wave- 2 topography simulations. Full model responses are shown by the squares. The crosses show the expected response if the stratification is held fixed. The solid line shows the expected response if the adiabatic heating is held fixed. (c) Maximum in the TEM overturning streamfunction at $70 \mathrm{hPa}$, and (d) vertical EP fluxes from $50^{\circ}$ to $90^{\circ} \mathrm{N}$ at $100 \mathrm{hPa}$, for both sets of simulations. Error bars indicate $95 \%$ confidence intervals for all quantities.

can then be computed assuming the adiabatic heating remains unchanged from the control run:

$$
\left\langle T-T_{\mathrm{rad}}\right\rangle^{t}=-\frac{\left\langle S \bar{w}^{*}\right\rangle^{t}}{\alpha} \approx-\frac{\left\langle S \bar{w}^{*}\right\rangle_{c}^{t}}{\alpha} .
$$

Here the stratification is given by $S=H N^{2} / R$, where $N^{2}$ is the square of the buoyancy frequency, $H$ is the scale height, and $R$ is the dry gas constant. Time averages of a quantity are indicated by $\langle\cdot\rangle^{t}$, and the subscript $c$ indicates the quantity is computed from the control run.

Figure 4 a shows the polar cap-averaged $\left(60^{\circ}-90^{\circ} \mathrm{N}\right)$ temperatures at $100 \mathrm{hPa}$ for the wave- 1 simulations. The full response is indicated by the squares. The solid line shows the temperature expected if the adiabatic heating is held fixed at the control run value for this region. The polar cap temperatures for the weakest damping runs do not increase as much as expected by this direct effect as a result of the weakened overturning circulation. To give a sense of whether it is the change in circulation or the change in stratification that is driving the temperature change, the crosses show the temperatures expected if only the stratification is held fixed at the control run value $\langle S\rangle_{c}^{t}$. At the weakest (and strongest) damping rates, most of the dynamical feedback arises from the weakened residual circulation. There is a narrow parameter regime between $\alpha_{\mathrm{LS}}=0.25$ and 0.3 for which the enhanced vertical temperature gradients induced by the warming lead to a weak positive dynamical feedback.

Similar quantities are plotted in Fig. $4 \mathrm{~b}$ for the wave-2 topography simulations. Although the overall meridional circulation decreases with weakened damping, the polar warming here in fact exceeds the direct effect as a result of the circulation moving poleward into the vortex. As with the wave- 1 simulations, much of the dynamical feedback is attributable to changes in the circulation, though the enhanced stratification plays a nonnegligible role.

Figure $4 \mathrm{c}$ shows the maximum of the TEM streamfunction at $70 \mathrm{hPa}$ for both sets of simulations. For damping rates near the value of the control run, the wave-1 simulations have a stronger Brewer-Dobson circulation than do the wave- 2 simulations. At both extremes the opposite is true. In both cases the overturning circulation decreases with weakened damping.

The planetary-scale Eliassen-Palm (EP) flux at the base of the stratosphere is shown in Fig. 4d. Perhaps surprisingly, the wave- 2 fluxes are stronger than the wave- 1 fluxes for all values of the damping. In both cases the fluxes decrease with weakened damping for much of the parameter regime considered, though this does not hold for the wave- 1 run with the strongest damping. The dynamical response in both sets of runs therefore is an overall reduction in the wave driving of the stratosphere, rather than simply a rearrangement of where the momentum carried by the waves is deposited.

In summary, the dominant change in the time-averaged zonal-mean state can be understood through the direct radiative effect of the weakened lower-stratospheric damping rates. These require a larger polar cap temperature anomaly to balance the dynamically driven adiabatic warming, thereby resulting in a warmed lower stratosphere. Dynamical feedbacks do play a quantifiable 
role as well; in general the wave fluxes driving the Brewer-Dobson circulation weaken with the damping, though in the wave-2 runs the flux convergence moves poleward as well, resulting in a narrow region of enhanced downwelling over the pole.

\section{b. Tropospheric changes}

The time-mean, polar lower-stratospheric warming is very similar to that imposed in a similar model by Haigh et al. (2005) and in a subsequent related study (Simpson et al. 2009). In the present case, however, the warming is induced in the context of a polar vortex with more realistic dynamics and variability. As expected, this warming induces an equatorward shift in the tropospheric jets, which can be seen in Figs. 2 and 3. This shift involves both an equatorward shift in the surface winds and an enhanced vertical shear, in balance with an increased equator-to-pole temperature gradient on the equatorward side of the jet, and the reverse on the poleward side of the jet.

The shift in zonal-mean torques required to maintain the shift in surface winds against friction is usefully diagnosed by vertically integrating the angular momentum budget. In the terrain-following sigma coordinates used by the model, the relevant terms are (e.g., Laprise and Girard 1990)

$$
\begin{aligned}
& \frac{\partial}{\partial t}\left(\left[\overline{p_{s} \mathcal{L}}\right]\right)+\frac{1}{a \cos \phi} \frac{\partial}{\partial \phi}\left(\cos \phi\left[\overline{p_{s} v \mathcal{L}}\right]\right)-\overline{\Phi_{s} \partial_{\lambda} p_{s}} \\
& \quad-a \cos \phi\left[\overline{p_{s} \mathcal{F}}\right]=0 .
\end{aligned}
$$

Here $\mathcal{L}=a \cos \phi(u+a \Omega \cos \phi)$ is the angular momentum; $\mathcal{F}=-k u_{s}$ is the surface friction (the sponge-layer torques are neglected); $u$ and $v$ are the zonal and meridional winds, respectively; $p$ is the pressure; the subscript $s$ indicates a surface quantity; and the rest of the symbols are standard. Square brackets indicate the vertical integral $[\cdot]=\int_{0}^{1}(\cdot) d \sigma$, and overbars indicate a zonal mean. Rewriting these in terms of the surface wind,

$$
U=L_{t}+M+D
$$

where

$$
\begin{aligned}
U & =\frac{\overline{p_{s} u_{s}}}{p_{s 0}}, \quad L_{t}=-\frac{\partial}{\partial t}\left(\frac{\left[\overline{p_{s} \mathcal{L}}\right]}{a \cos \phi k \Delta \sigma p_{s 0}}\right), \\
M & =-\frac{1}{a^{2} \cos ^{2} \phi} \frac{\partial}{\partial \phi}\left(\frac{\cos \phi\left[\overline{p_{s} v \mathcal{L}}\right]}{k \Delta \sigma p_{s 0}}\right), \quad D=\frac{\overline{\Phi_{s} \partial_{\lambda} p_{s}}}{a \cos \phi k \Delta \sigma p_{s 0}} .
\end{aligned}
$$

Here $\Delta \sigma$ is the thickness of the surface layer in the model and $p_{s 0}$ is a reference surface pressure, taken here to be $1000 \mathrm{hPa}$. The transient term is then neglected in the time mean.

The time-mean balance for the two control runs is shown in Figs. 5a and 5b. The surface wind produced by the model is shown by the thick solid line, while the wind predicted by Eq. (4) is shown by the thin solid line. In all runs, the surface westerlies are maintained by angular momentum transport, but with the form $\operatorname{drag} D$ constituting a large component of the balance in the Northern Hemisphere.

The net effect of the topography can be seen as the sum of the momentum flux convergence due to the topographically generated waves as well as the form drag. This separation is straightforward in the present runs as a result of the sinusoidal topography used. Assuming that planetary waves generated by wave-wave interactions between the baroclinic eddies can be neglected (this is supported by the absence of any such momentum flux convergence in the Southern Hemisphere balance), the momentum flux convergence can be decomposed into that generated by the two gravest zonal wavenumbers $M_{1,2}$ and the rest, which will be dominated by the synoptic scales $M_{s}=M-M_{1,2}$. The net forcing by the topography is then given by $M_{p}=D+M_{1,2}$. These two terms are shown with the thick dashed lines in Fig. 5. The synoptic-scale momentum fluxes in each hemisphere in the wave-1 control are in fact quite similar; the difference in the total momentum flux convergence arises from the contribution of the planetary scales. This additional momentum flux convergence is, however, more than compensated for by the form drag, which explains the weaker surface westerlies in the Northern Hemisphere.

The planetary-scale momentum fluxes are considerably stronger in the wave- 2 control run than in the wave- 1 control run, as is also implied by Fig. $4 d$. The Northern Hemisphere synoptic-scale fluxes are also stronger, but the sum is balanced by a much enhanced form drag. The changes in synoptic-scale fluxes between Figs. 5a and 5b are consistent with the change expected (Haigh et al. 2005) from the decreased uppertropospheric temperatures in the wave- 2 control run (with respect to the wave-1 control). Note, however, that the shift in the surface winds between these runs does not project onto the annular mode (the center of the jet weakens and both of its flanks accelerate; not shown).

The time-averaged change induced by the weakened damping rates is shown in Figs. $5 \mathrm{c}$ and $5 \mathrm{~d}$. In both cases and both hemispheres the response of the surface wind is dipolar, corresponding to an equatorward shift that would project strongly onto the annular mode. The synoptic-scale fluxes weaken, resulting in 

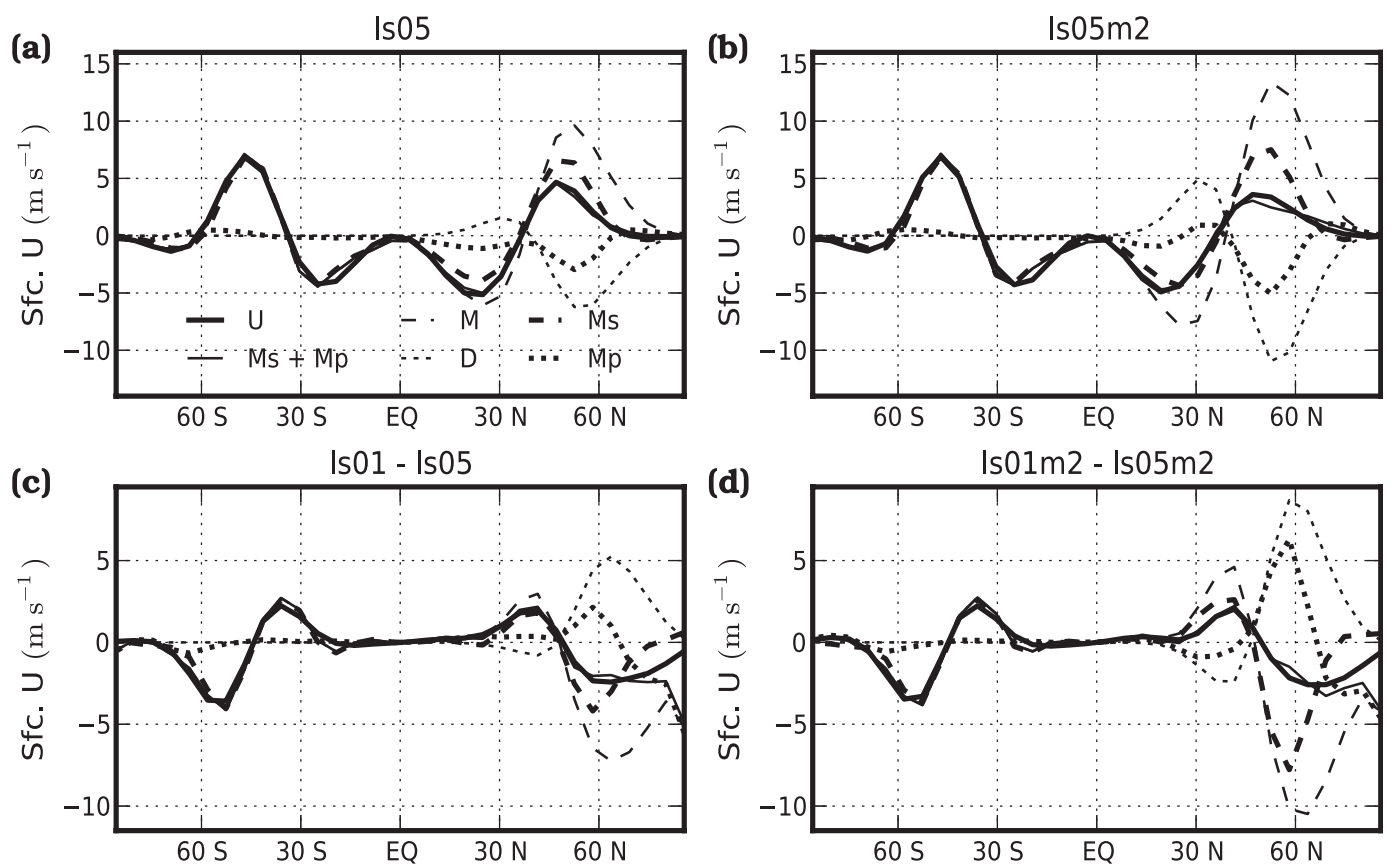

FIG. 5. Vertically integrated, zonal-mean angular momentum budget, shown in units of the surface wind (see text) for (a) wave-1 and (b) wave-2 control runs and the change seen in (c) wave-1 and (d) wave-2 weakened damping run. In all cases, the solid thick line shows the zonal-mean surface wind, while the solid thin line is the surface wind implied by the balance. The budget is subdivided in two ways: into the total momentum flux convergence (thin dashed line) and the form drag (thin dotted line), and into the synoptic-scale momentum flux convergence (thick dashed line) and the sum of the form drag and the planetary-scale flux convergence (thick dotted line).

more convergence on the equatorward flank of the jet and less on the poleward flank. This change in synopticscale eddies is consistent with that found by other studies (e.g., Song and Robinson 2004; Kushner and Polvani 2004; Simpson et al. 2009). The synoptic-scale response is nearly the same in both hemispheres in the wave-1 runs, while the response (particularly on the poleward side of the jet) is considerably stronger in the Northern Hemisphere in the wave- 2 runs. The net effect of the topography is to counteract the shift in the synoptic-scale momentum fluxes: although the planetary-scale fluxes reinforce the changes induced by the synoptic-scale eddies, the increased form drag again more than compensates. This effect is also stronger in the wave- 2 runs than it is in the wave- 1 runs, such that the change in the surface winds in both sets of simulations is approximately the same. This is consistent with the tendency for the tropospheric jet to be collocated with the topography, and for the annular mode decorrelation time scales to be reduced in the presence of topography (Gerber and Polvani 2009). However, examination of the lag correlation between the shifting of the jets and the terms $M_{s}$ and $D$ suggests that the reduction in this case arises from the direct effect of the form drag on the surface winds, rather than by disrupting the spatial correlation of the smaller-scale eddies as was found by Gerber and Polvani (2009). This may be attributable to the difference in the zonal scales of the imposed topography.

Note that although the net effect of the topography is to counteract the surface wind response, the reduced planetary-scale heat fluxes are in part balanced by anomalous diabatic heating where these fluxes diverge or converge. In the present setup where the diabatic heating is given by Newtonian cooling, this implies a change in the meridional temperature gradient and a corresponding change in the shear. As can be seen in Figs. 2 and 3, the sign of the shear is the same as that of the underlying changes in the surface wind, resulting in a larger upper-tropospheric signal. Moreover, since the tropospheric damping rates are the same in all experiments, the larger change in fluxes in the wave- 2 simulations implies the larger tropospheric temperature anomalies found therein.

While the stratospheric reduction in planetary wave fluxes might best be understood through CharneyDrazin-type filtering as a result of the weakened zonal winds, it is less clear why the planetary-scale fluxes should also weaken in the troposphere. The likely candidates are either enhanced reflection, or reduced generation. This question is considered further below. 


\section{Variability}

\section{a. Abacus plots}

To analyze the stratospheric variability in these runs, two types of indices are used: that of the NAM and of the PJO. The indices of the NAM are computed from zonalmean geopotential heights following Gerber et al. (2010). The global mean is removed at each day, and the zonal-mean anomalies north of the equator are area weighted prior to computing the first EOF of the geopotential height anomalies. The corresponding principal component time series at each pressure level is then used as the NAM index at that level.

The PJO is indexed here by the first two EOFs of the polar cap-averaged $\left(70^{\circ}-90^{\circ} \mathrm{N}\right)$ temperature anomalies. In observations (Kuroda and Kodera 2004) and in comprehensive chemistry-climate models (Hitchcock et al. 2013), these modes describe deep vertical dipolar temperature anomalies. In the sign convention adopted here, the first mode describes a warming of the upper stratosphere and a coincident cooling of the mesosphere, while the second describes a warming of the lower stratosphere and a cooling in the upper stratosphere. These two modes collectively describe roughly $90 \%$ of the total (deseasonalized) polar cap-averaged temperature variance. The structure of these EOFs in a run equivalent to the wave- 1 topography control run has been shown in Kohma et al. (2010), and agrees well with the calculations shown here. The vertical structure of these EOFs in the control runs and weakened damping runs is shown in Fig. 6. Emphasis is placed on the structure up to the upper stratosphere as the details in the mesosphere will be affected by the use of Rayleigh friction as a crude gravity wave drag parameterization above $1 \mathrm{hPa}$.

Figure 6a shows the first and second EOFs for the wave- 1 control run and the weakened damping run. As in the observations, the first mode describes upperstratospheric anomalies, while the second mode captures lower-stratospheric anomalies. In the weakened damping run, the amplitude of the first mode has decreased somewhat, the amplitude of the second has increased, and the lower-stratospheric maximum has shifted downward. Figure $6 \mathrm{~b}$ shows the same EOFs for the wave-2 topography. The EOFs of the control run have much weaker amplitudes in the lower stratosphere, which is consistent with the reduced wave driving at high latitudes in this run (cf. Figs. 2c, 3c). The EOFs of the weakened damping run, however, agree well with those of the weakened damping wave-1 run.

The fraction of the variance explained by the two modes as a function of the lower-stratospheric damping rate is summarized in Fig. 6c. The fraction explained by
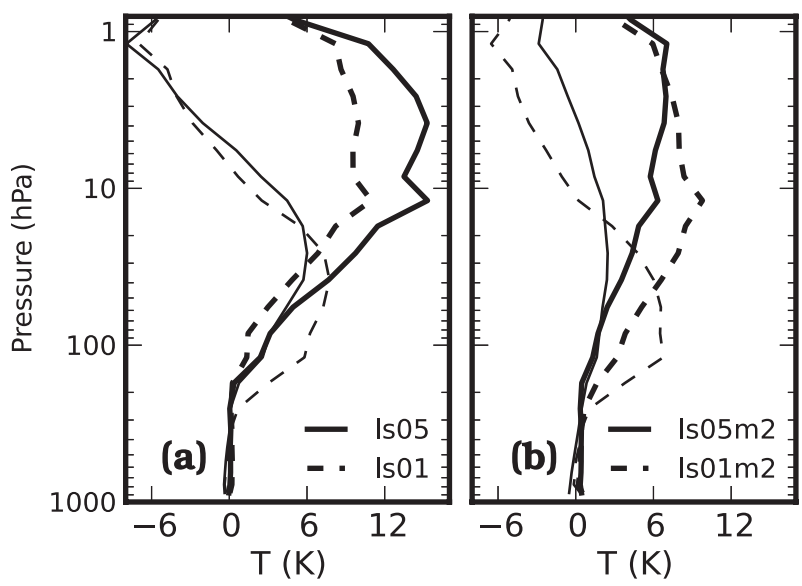

(c)

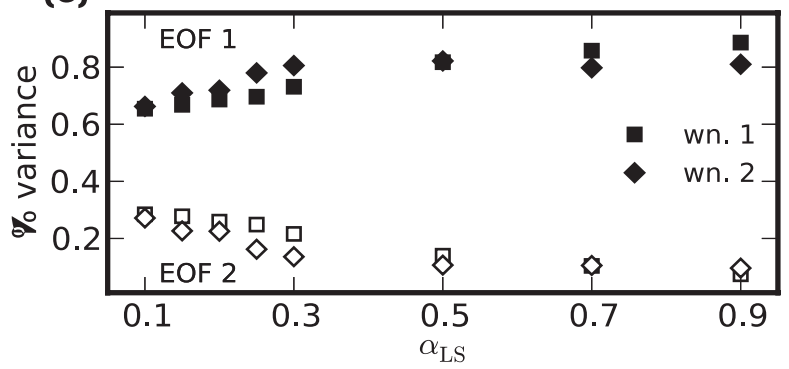

FIG. 6. The first two EOFs of polar cap-averaged temperature anomalies for the control run (solid lines) and a weakened damping run (dashed lines) for the (a) wave- 1 and (b) wave- 2 simulations. The first EOF is indicated by the thick lines, and the second by the thin lines. (c) Fraction of variance explained by the first (filled symbols) and second (open symbols) EOF.

the first mode is indicated by the filled symbols for all runs, while that explained by the second is indicated by the open symbols. For both sets of simulations, the fraction explained by the second EOF increases as the damping rate decreases, again consistent with a more variable lower polar stratosphere, with the second mode describing a somewhat higher fraction in the wave-1 simulations than in the wave- 2 simulations. The total fraction of variance explained by the two modes is roughly constant across all runs.

The principal component (PC) time series $\mathrm{ts}_{1}$ and $\mathrm{ts}_{2}$ of these two modes are not dynamically independent. Trajectories in the two-dimensional phase space defined by these two modes typically rotate counterclockwise, corresponding to downward propagation of temperature anomalies. The shift in variance toward the second $\mathrm{EOF}$ as the lower-stratospheric damping is weakened is consistent with these anomalies propagating deeper into the lower stratosphere. Following Hitchcock et al. (2013), the trajectory can be transformed to polar coordinates $r$ and $\theta$, defined by $r^{2}=\mathrm{ts}_{1}^{2}+\mathrm{ts}_{2}^{2}$ and $\tan \theta=\mathrm{ts}_{2} / \mathrm{ts}_{1}$. The trajectories can then be visualized using "abacus" 

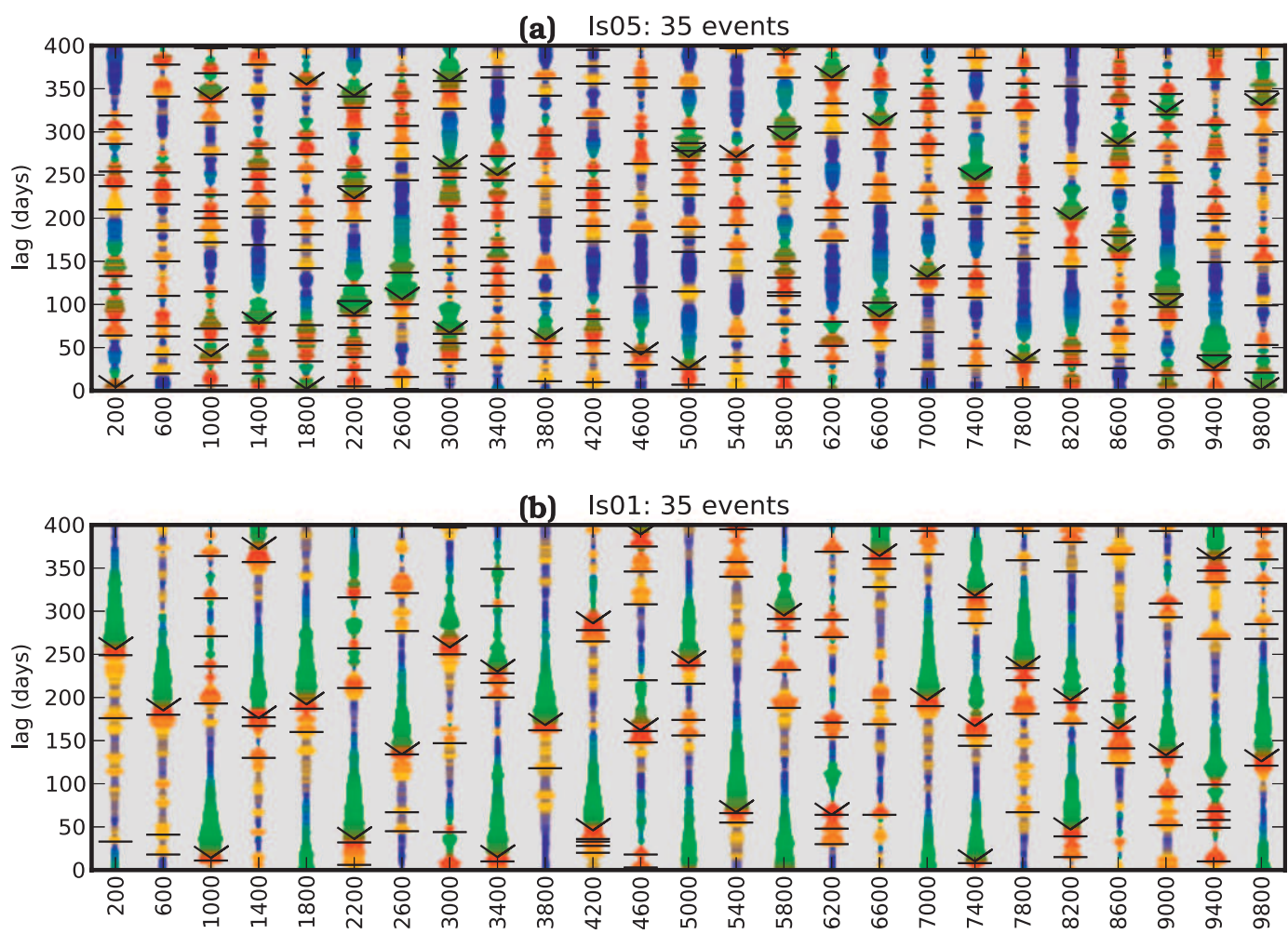

FIG. 7. Abacus plots (see text) for wave-1 (a) control run and (b) weakened damping run. Weak vortex events (as defined by the annular mode index at $10 \mathrm{hPa}$ ) are indicated by chevrons. The number of such events in each run is given in each panel title. Pulses of EP flux convergence are shown by the horizontal lines.

plots: the time series are represented as a vertical ribbon which has a width proportional to $r$ and is colored according to $\theta$. The color is determined as follows: When $\mathrm{ts}_{1}$ is positive $(\theta=0)$ the ribbon is red, when $\mathrm{ts}_{2}$ is positive $(\theta=\pi / 2)$ the ribbon is green, when $\mathrm{ts}_{1}$ is negative $(\theta=\pi)$ the ribbon is blue, and when $\mathrm{ts}_{2}$ is negative $(\theta=$ $3 \pi / 2)$ the ribbon is yellow. Intermediate phases are interpolated in red-green-blue (RGB) space. For more discussion and examples of these plots, see Hitchcock et al. (2013). Rather than use the PC time series computed directly from each run, the anomalies are projected on to the EOFs of the wave- 1 control run in order to more directly compare the behavior in different runs, which is the primary purpose of this section. Specifically, this choice makes evident the weaker variability in the wave- 2 control run below.

Abacus plots for the wave- 1 control run and weakened damping run are shown in Figs. $7 \mathrm{a}$ and $7 \mathrm{~b}$, respectively, for days 200-10 200. Each vertical ribbon corresponds to 400 days of model time, which runs up and to the right in these plots. Also shown (chevrons) are the dates of weak vortex events as computed from the NAM index at $10 \mathrm{hPa}$ following McLandress and Shepherd (2009). A threshold of $-2.5 \sigma$ is used here.
Note that this results in a relatively constant number of events being identified in each run, despite the fact that runs with stronger damping generate substantially weaker events. This is explored further below.

The difference in the character of the variability in these two runs is revealed by the abacus plots. In the control run, the vortex is much more frequently disturbed by minor warmings (apparent as red and green pulses) with time scales of about 20 days. While more stable periods do occur (following weak vortex events near days 6700,9100 , and 9400 , for instance), these are the exception, not the rule. In contrast, the weakened damping run shows a very regular, long time-scale response to the weak vortex events, evidenced by the slowly narrowing ribbons following the chevrons, which slowly shift from red to green to blue. These events are strongly reminiscent of the PJO events identified by Hitchcock et al. (2013). While there are some periods characterized by shorter time-scale events (days 62006900 , for instance), again the dominant behavior is that of the long time-scale recoveries.

The relationship of these modes of variability to the dynamical driving of the vortex by planetary waves is also shown on these plots. The horizontal black lines indicate 
(a) Is05m2: 27 events

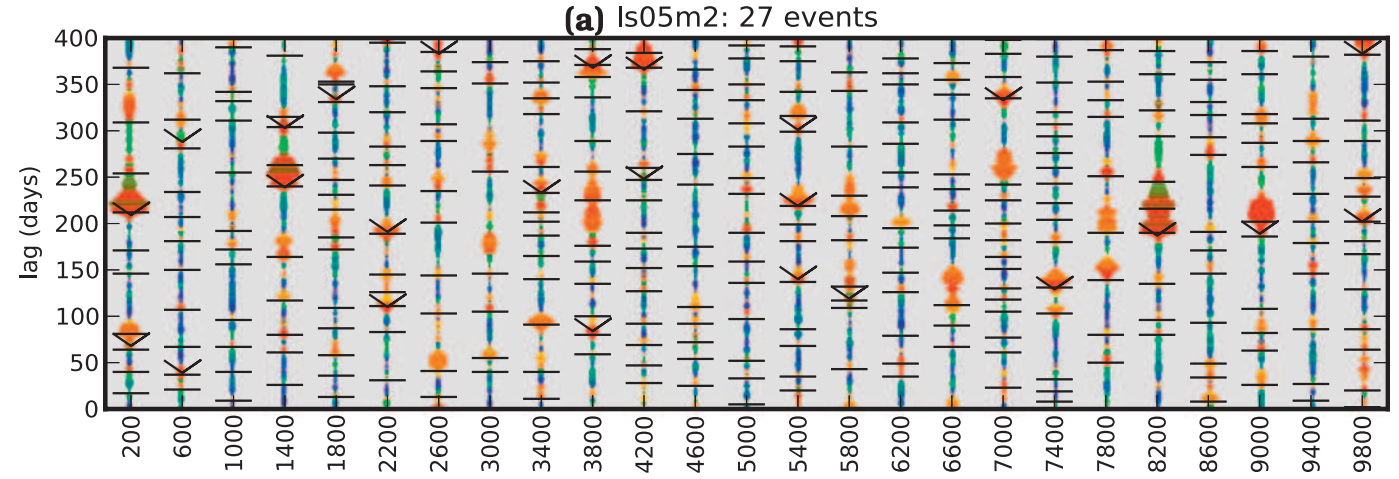

(b) Is01m2: 25 events

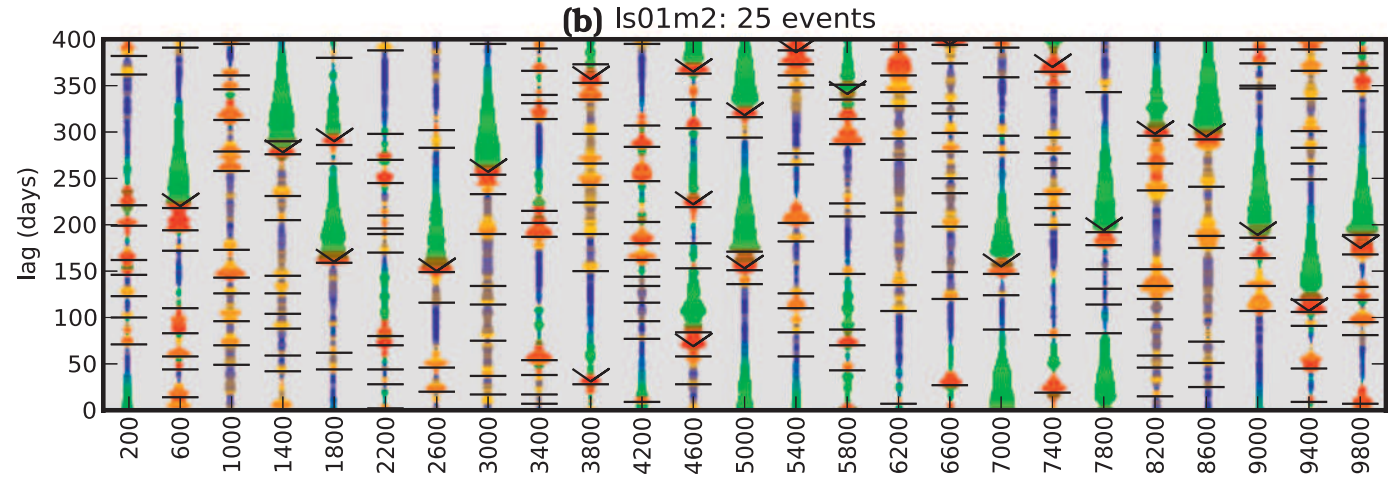

FIG. 8. As in Fig. 7, but for the wave 2 simulations.

local maxima in the total EP flux convergence averaged between 10 and $1 \mathrm{hPa}$, from $50^{\circ}$ to $90^{\circ} \mathrm{N}$. The time series has been smoothed by a 15-day low-pass filter, and only maxima corresponding to a deceleration of more than $7 \mathrm{~m} \mathrm{~s}^{-1}$ day $^{-1}$ are shown. These pulses drive a warming of the upper stratosphere, as can be seen by the tendency of the ribbon to widen and become redder following the pulses. They occur much more frequently in the control run and are clearly suppressed following the weak vortex events in the weakened damping run.

Similar abacus plots are shown for the wave-2 topography runs in Fig. 8. In this case variability of any kind is clearly suppressed in the wave- 2 control run. The weak vortex events are strongly confined to the upper stratosphere (almost no positive anomalies of the second mode are apparent), and the induced anomalies do not persist for longer than about a month. The lack of sudden warmings in runs with wave- 2 topography at this horizontal resolution has been reported by Taguchi et al. (2001) and Gerber and Polvani (2009). In the weakened damping run, however, the zonal-mean structure of the weak vortex events closely resembles that of the events in the wave- 1 weakened damping run.

Since the wave driving in the upper stratosphere is generally weaker in the wave- 2 runs than in the wave- 1 runs, we consider a lower threshold for the magnitude of the local maxima ( $3 \mathrm{~m} \mathrm{~s}^{-1}$ day $^{-1}$ instead of $7 \mathrm{~m} \mathrm{~s}^{-1}$ day $^{-1}$ used above). The same selective suppression during the extended time-scale recoveries is apparent in the wave- 2 weakened damping run.

\section{b. Stratospheric composites}

To further demonstrate the suppression of the EP flux, Fig. 9 shows composites of the zonal-mean zonal wind at $60^{\circ} \mathrm{N}$ (contour lines) and of the vertical EP fluxes from $50^{\circ}$ to $90^{\circ} \mathrm{N}$ (filled contours) following weak vortex events in these four runs. The filled contours are faded where they do not differ significantly from climatology at the $95 \%$ confidence interval. The zero wind line is thick. The effects of the weakened damping rates on the warming events are twofold. First, reduced damping allows a deceleration induced by the same eddy fluxes to descend farther in the stratosphere, since the relaxational diabatic heating rates are weaker. Second, once the circulation is disrupted in the lower stratosphere, the anomaly can persist for longer assuming some further dynamical activity does not perturb it again. Both of these effects can be seen in the wind contours in Fig. 9. Inspection of the $20 \mathrm{~m} \mathrm{~s}^{-1}$ contour shows that it descends only to $10 \mathrm{hPa}$ in the wave- 1 control run, but it descends nearly to $100 \mathrm{hPa}$ in the weakened damping run, and extends out nearly 60 days following the central date. 

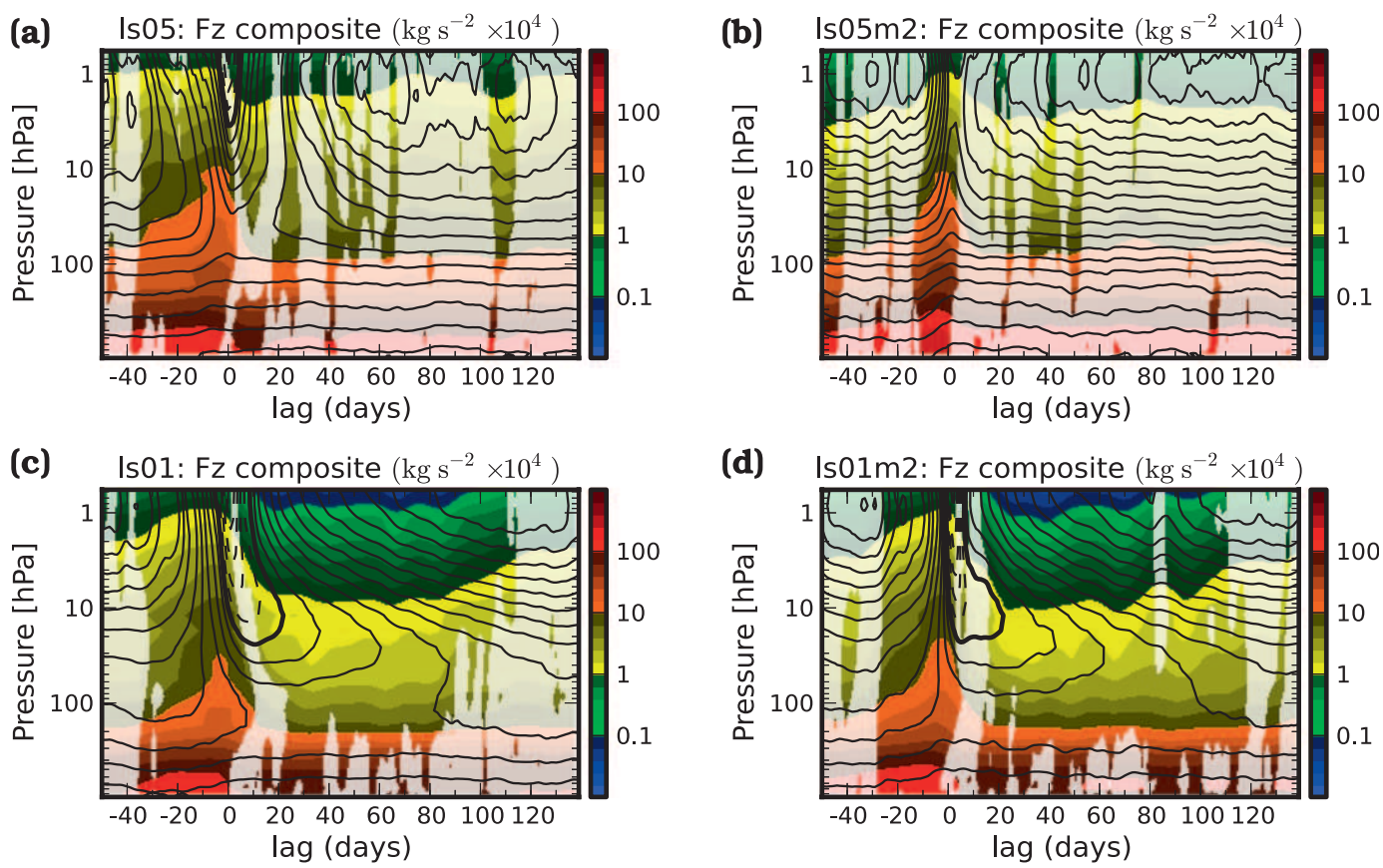

FIG. 9. Composites of weak vortex events in the (a) wave-1 and (b) wave- 2 control runs and in the (c) wave-1 and (d) wave- 2 weakened damping runs. Contour lines indicate the absolute zonal-mean zonal wind at $60^{\circ} \mathrm{N}$ at an interval of $10 \mathrm{~m} \mathrm{~s}^{-1}$. The zero wind line is thick. Filled contours indicate the absolute vertical EP flux from $50^{\circ}$ to $90^{\circ} \mathrm{N}$ in $\mathrm{kg} \mathrm{s}^{-2}$. The contours are spaced logarithmically. For the filled contours, periods that are not statistically significant at the $95 \%$ confidence level are partially masked in white.

Similar behavior is seen in the wave- 2 weakened damping run, though the events in the control run are so weak that the $20 \mathrm{~m} \mathrm{~s}^{-1}$ contour is hard to distinguish.

The vertical fluxes shown are the total fluxes, to better compare the four runs. The enhanced fluxes that trigger the event are apparent in all four runs. They are somewhat weaker in the weakened damping runs relative to the control and are in fact strongest at the base of the stratosphere in the wave- 2 control, as is the case with the time-averaged flux shown in Fig. 4d. Most importantly, the strong suppression following the events in the two weakened damping runs is clear. The suppression extends all the way down to the surface, so it is not a matter of the waves simply being refracted equatorward. This is consistent with the suppression seen following PJO events in a comprehensive model (Hitchcock et al. 2013). The suppression is clearly coincident with the weakened lower-stratospheric winds, though the absence of a zero wind line for much of this period precludes the direct application of the Charney-Drazin criterion as an explanation. Calculations with a linear, steady-state stationary wave model (Harnik and Lindzen 2001) forced by the same topography do suggest that the anomalous zonal-mean circulation in the lower stratosphere can explain roughly $50 \%$ of the reduction in upward EP flux, with the majority of this reduction arising from the reduced potential vorticity gradient (not shown). The tropospheric fluxes are not suppressed in the linear model. A more complete understanding of this suppression, though clearly desirable, is beyond the scope of the present study.

\section{c. Tropospheric response}

The wind and temperature anomalies in the lowermost stratosphere also induce an equatorward shift in the tropospheric jets, as in the time-averaged response. Composites of the tropospheric zonal wind anomalies at $300 \mathrm{hPa}$ are shown (filled contours) in Fig. 10 for the same four runs. The difference between the temperature anomalies at 100 and $300 \mathrm{hPa}$ is also shown (contour lines) as a proxy for the anomalous upper-tropospheric static stability. As in Fig. 9, the filled contours are faded where the zonal winds do not differ significantly from the climatology. The equatorward shift in the jet coincides closely with the enhanced upper-tropospheric stability at high latitudes. It is much more persistent in the weakened damping runs than it is in either of the control runs. The magnitude of the shift, however, depends more strongly on the wavenumber of the topography than it does on the duration of the event. Moreover, there is a significant shift even in the wave-2 control run events, which do induce a brief period of 

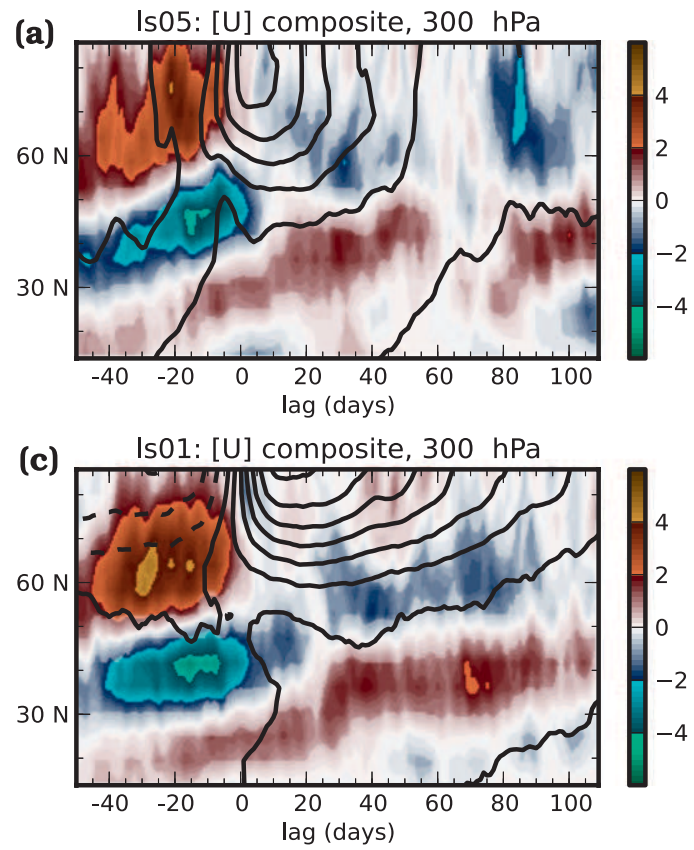
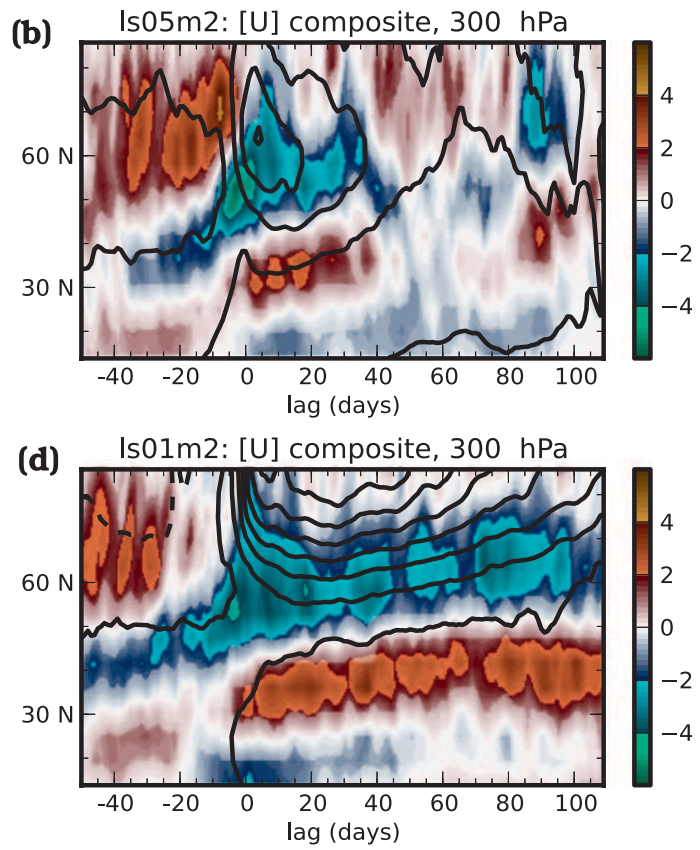

FIG. 10. As in Fig. 9, but for the difference in zonal-mean temperatures between 300 and $100 \mathrm{hPa}$ (contour lines; interval of $2 \mathrm{~K}$ ) and zonal-mean zonal wind anomalies at $300 \mathrm{hPa}$ (shaded contours; $\mathrm{m} \mathrm{s}^{-1}$ ).

enhanced stability in the upper troposphere despite the very weak stratospheric variability. As expected from the stronger equatorward refraction of wave- 2 planetary waves, this enhanced stability is at a lower latitude than in the other runs. Note that while this strong correlation between the upper-tropospheric stability and the shift in the tropospheric jet is consistent with, for instance, Simpson et al. (2009), changes in the lowerstratospheric winds may also be playing an important role.

A very strong tropospheric precursor to the warmings is also seen in all four runs, in which the jets shift poleward during the period of enhanced upward EP fluxes. This has been noted in some studies (Cohen and Jones 2011), though the phenomenon seems especially strong in these runs. Curiously, this precursor strengthens in the wave-1 weakened damping run (with respect to the control), but it weakens in the wave- 2 weakened damping run. Further investigation of this is left for future study. The correspondence between the tropospheric jet location and the enhanced wave fluxes does raise the possibility that the generation of planetary waves may be affected by the latitude of the jet. This would provide a mechanism for a tropospheric feedback on the stratospheric time scales: the equatorward shift of the jet induced by the upper-tropospheric anomaly would in turn reduce wave generation, permitting the anomaly to persist. This feedback cannot be the primary determinant of the stratospheric time scales given the similar magnitudes of the tropospheric shift in the control runs, but it may serve to extend them.

The terms responsible for the redistribution of angular momentum associated with the tropospheric jet shift are considered in Fig. 11 in more detail. Here composites of the terms in the budget described by Eq. (4) are shown for the two weakened damping runs, in which the tropospheric response is most persistent. To improve the statistics, we have considered the 100 200-day runs. The shorter 10 200-day runs show a similar response, but

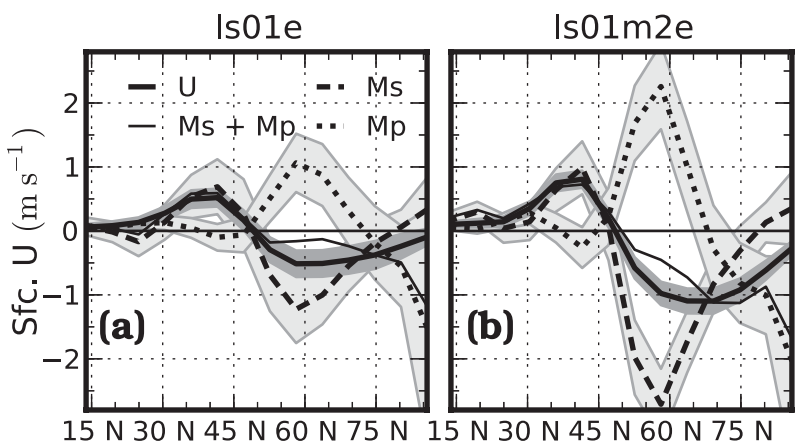

FIG. 11. Composited anomalies in the vertically integrated angular momentum budget for (a) wave-1 and (b) wave- 2 weakened damping experiments. Lags of 30-90 days from the central event are used for (a) and 5-100 days for (b). Terms are labeled as in Fig. 5. Shading shows the $95 \%$ confidence interval for the surface wind anomaly (thick solid line) and $90 \%$ confidence intervals for the synoptic- and planetary-scale momentum flux convergence terms (dashed and dotted lines). 
the uncertainties are considerably larger. The terms are time averaged over the period during which the jet has shifted in the composite: lags of 30-90 days following the central date for the wave- 1 experiment, and lags of 5-100 days for the wave-2 experiment. Estimates of the $95 \%$ confidence interval for the surface wind response and for the two flux convergence terms are shown. The confidence interval for the surface wind response estimated by the budget is omitted for clarity; note however that despite the relatively large uncertainties in this sum, the response in both cases is well predicted by the assumption of steady state. This is consistent with the steadiness of the response seen in the composites; the transient term $L_{t}$ can thus be neglected. The jet shift is sustained by a persistent change in the synoptic-scale eddies, which is consistent with the dynamics of the annular mode. The net effect of the form drag and planetary-scale momentum fluxes is to counteract this response.

The structure of the transient response shown in Fig. 11 is very similar to that of the time-mean response shown in Figs. 5c and 5d. Even if there are feedbacks between the troposphere and the stratosphere that prolong the stratospheric anomaly, it suggests that the dynamics of the shift in the jet following a PJO event are fundamentally similar to those driving the time-mean response. If this is in fact the case, it supports the consideration of the shift in the tropospheric jets during sudden warmings as a response to an external forcing (Keeley et al. 2009), rather than a fully coupled, dynamically distinct response.

\section{d. Annular mode time scales}

The vertical profile of NAM time scales is shown for a subset of the wave-1 and wave- 2 experiments in Fig. 12. A $95 \%$ confidence interval is included on the weakest damping run in each case for reference; see the appendix for details of how these time scales and confidence intervals are estimated. Somewhat unexpectedly, very little sensitivity to the radiative damping time scales is seen in the stratospheric annular mode time scales in the wave- 1 topography runs, despite the clear difference in the character of the variability seen in Fig. 7 and in composites shown below. Closer inspection of the principal component time series at stratospheric levels suggests that these decorrelation time scales correspond to different physical processes as the damping time scale is varied. For the weakest damping runs, this time scale does correspond roughly to the decay time scale of the large PJO-type events apparent in Fig. 7b. For stronger damping runs, however, inspection of the NAM time series suggests that the decorrelation time scale corresponds qualitatively to the typical time between large anomalies, despite

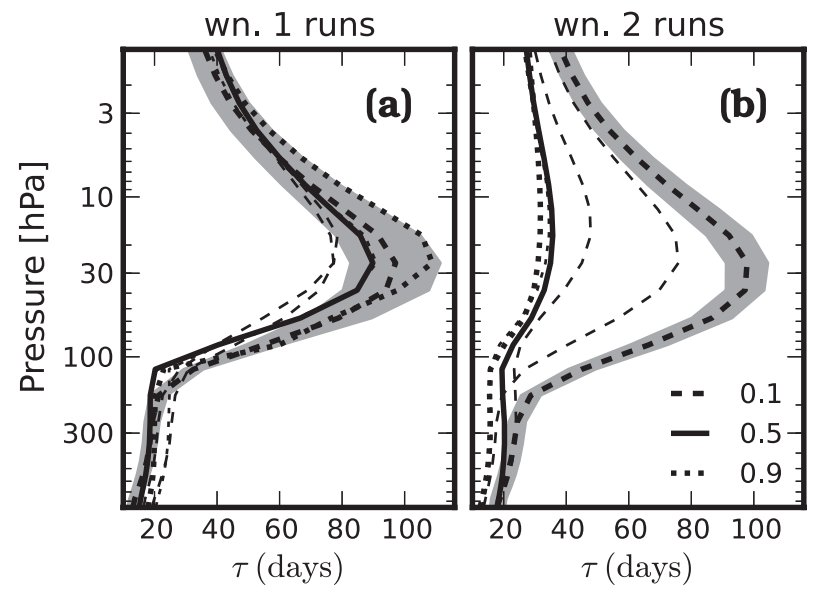

FIG. 12. Vertical profile of annular mode time scales in the Northern Hemisphere for (a) wave-1 and (b) wave-2 topography runs. The weakest and strongest damping runs are labeled in the legend, as is the control run for both cases; also shown are runs with $\alpha_{\mathrm{LS}}=0.2,0.3$ (thin dashed), and 0.7 (thin dotted). The gray shading in both panels indicates the $95 \%$ confidence interval for the weakest-damping-run time scales.

the relatively rapid decorrelation of the large anomalies themselves (not shown). In contrast, the wave- 2 runs exhibit the expected annular mode time-scale profiles, which correspond closely to the radiative damping time scales in the stratosphere. The time scales saturate for the stronger damping runs, which may explain the lack of sensitivity seen by CO. The difference in behavior between the wave- 1 and wave- 2 runs may correspond to the relatively weak zonal-mean variability exhibited by the wave-2 control run (Fig. 8a).

The tropospheric NAM time scales in all cases are relatively realistic and are comparable to those reported by Gerber et al. (2008). There is a weak suggestion of sensitivity to the radiative damping in the wave- 2 runs, but it is not strong enough to rise above the sampling uncertainty, with at least one of the weaker damping runs exhibiting similar annular mode time scales to the strongest damping run. They do not show any clear sensitivity to the jet location (see also below), which is in contrast to recent expectations (Kidston and Gerber 2010) and to what one might expect based on the enhanced persistence of the composited response to stratospheric events seen in Fig. 10.

\section{e. Summary of transient response}

The sensitivity of the variability in these runs to the strength of the lower-stratospheric radiative damping described in detail above is summarized in Fig. 13. Figure $13 \mathrm{a}$ shows the maximum pressure to which the $5 \mathrm{~m} \mathrm{~s}^{-1}$ contour descends during the weak vortex events as a function of the lower-stratospheric damping time-scale 

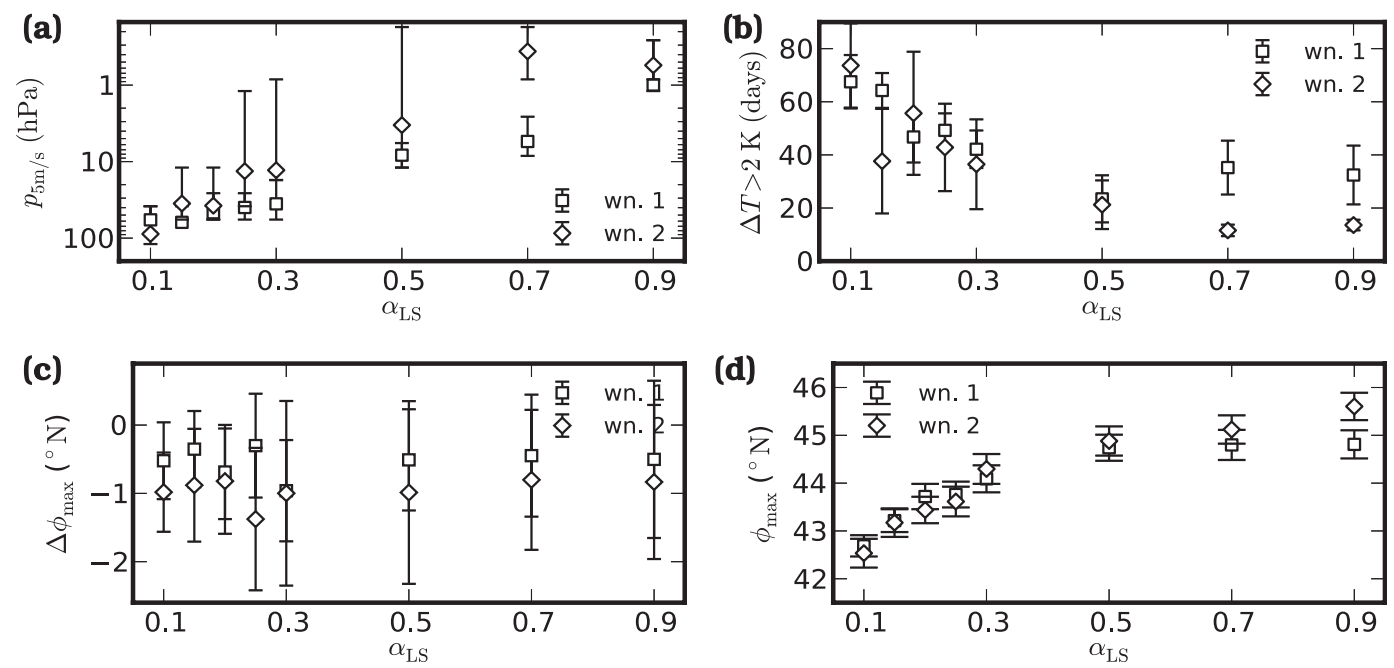

FIG. 13. Composites of the weak vortex events in all runs: (a) maximum pressure to which the $5 \mathrm{~m} \mathrm{~s}^{-1}$ contour descends; (b) duration of the enhanced upper-tropospheric/lower-stratospheric stability, as indicated by the difference in polar cap-averaged $\left(70^{\circ}-90^{\circ} \mathrm{N}\right)$ temperature anomalies at 300 and $100 \mathrm{hPa}$ being greater than $2 \mathrm{~K}$; and (c) shift $\left(^{\circ}\right)$ of the latitude of the wind maximum at $300 \mathrm{hPa}$. (d) Time-averaged position of the jet maximum at $300 \mathrm{hPa}$.

parameter. The warmings descend more deeply into the lower stratosphere as the damping is weakened. The wave- 1 events tend to descend more deeply than do the wave- 2 events for all the runs except the weakest damping run, which is consistent with the greater tendency for wave- 2 planetary waves to be refracted equatorward. As the radiative time scales lengthen and the depth to which the warming descends approaches the tropopause, so too does the duration of the enhanced upper-tropospheric static stability (Fig. 13b). This sensitivity is only resolved for damping weaker than the control profile. Coincident with this period of enhanced upper-tropospheric stability, the tropospheric jets shift equatorward. As noted above, however, the magnitude of the shift (as opposed to its duration) is quite insensitive to the radiative damping time scales (Fig. 13c; the maximum is computed after interpolating the wind profile onto a $0.02^{\circ}$ grid). If anything, the magnitude is more strongly determined in the present runs by the wavenumber of the imposed topography. In contrast, the time-mean position of the jet varies strongly with the damping time scale, shifting by upward of $2^{\circ}$ poleward from the control run to the weakest damping run (Fig. 13d). It may be that the sensitivity of the transient response is simply too weak to be clearly resolved with the present statistics. One possible explanation for the weak sensitivity in the transient case is competing effects: while the stability changes induced by the warmings are stronger in the weaker damping runs, they are generally centered above the pole, and are thus farther away from the jet (which has moved equatorward). While the amplitude of the forcing may be increasing, its projection onto the annular models may be decreasing, resulting in a response whose magnitude remains roughly constant as the jet latitude shifts.

\section{Discussion and conclusions}

The present study has focused on the effects of the lower-stratospheric radiative damping on the variability of the stratospheric vortex and its coupling to the troposphere in a simplified global circulation model. The central result is that the character of the sudden warmings produced by the model changes drastically as a function of the radiative damping time scales. Weakening the radiative damping results in warmings that disrupt the vortex lower in the stratosphere, and that persist for longer. With a radiative damping profile that most closely matches that computed empirically from CMAM (Hitchcock et al. 2010), the character of the warmings becomes closely analogous to the PJO events identified by Hitchcock et al. (2013). In this respect our results differ from those obtained by the closely related study of $\mathrm{CO}$, who saw relatively weak sensitivity of the variability to stratospheric radiative time scales. Some of this disagreement can likely be attributed to the fact that the most sensitive response found here was for damping profiles that were substantially weaker than any considered by $\mathrm{CO}$ (see Fig. 13), though the difficulties in interpreting the decorrelation time scales of annular mode indices discussed in section $4 \mathrm{~d}$ may also be relevant.

Although the modified damping rates do affect the climatology of both the troposphere and the stratosphere, the changes in the stratosphere are dominated 
by the direct radiative response. By comparison, the timemean change in the wave driving of the stratosphere is relatively weak. It is therefore unlikely that the drastic changes in the variability of the wave driving are responding strongly to the steady-state changes in the zonal-mean circulation. The most likely interpretation is that the variability of the planetary waves is responding to (and determining) the change in the variability of the stratospheric zonal-mean state. The cause of the change in character of the variability is, by experimental design, ultimately stratospheric in origin, suggesting that the stratosphere does play an important role in mediating the waves responsible for its variability. Even if the statistics of the stratospheric waves are responding to some change in their generation by the tropospheric flow [as in the slaved-stratospheric variability paradigm of Plumb and Semeniuk (2003)] this change must still ultimately be influenced by some aspect of the stratospheric circulation, since only the stratospheric damping has been modified.

The structure of the stratospheric circulation anomalies initially produced by a sudden warming influences the subsequent evolution of the circulation in two ways. First, during the extended recovery period following the deepest warmings, further dynamical forcing is strongly suppressed. This is consistent with the behavior noted by Hitchcock et al. (2013) in the real atmosphere and in a more realistic model integration. That it is observed in the runs presented here confirms that this suppression is likely a response to the zonal-mean state of the stratosphere. The long stratospheric recovery is therefore predominantly radiative (Hitchcock and Shepherd 2013)—all else being equal, stronger damping would result in a more rapid return to climatological conditions. The extended recovery is, however, due as much to the extended absence of eddies as it is to the radiative time scales. The most natural hypothesis is that this absence is due to the weakened westerlies in the lower stratosphere. However, a steady, linear wave model can only explain a fraction of the suppression, and this primarily as a result of the weakened potential vorticity gradient. If the weakened potential vorticity gradient is in fact responsible, this may suggest that the reduced planetary-scale fluxes in the troposphere are due to enhanced reflection. Note that the vortex during the recovery is in a strongly absorbent configuration according to the index of Perlwitz and Harnik (2004) and Harnik (2009), with strong positive vertical shear throughout the polar stratosphere. Another possible effect suggested by the present experiments is that the equatorward shift of the tropospheric jet induced by the warming in turn reduces the generation of planetary waves. This latter effect, however, cannot be the whole story, since the magnitude of the tropospheric jet shift is quite similar in all of the present runs, while the persistence of the lower-stratospheric anomaly varies substantially.

The second effect of the lower-stratospheric warming is, as just mentioned, to induce an equatorward shift in the tropospheric jets. The duration of the shift is strongly correlated with the duration of the lower-stratospheric warming above. The change in surface friction produced by the shift in surface winds is in balance with the form drag and momentum flux convergence (consistent with the rapid adjustment period seen in Figs. 10c and d), and is driven by a shift in the synoptic-scale momentum flux convergences. The form drag and planetary-scale momentum flux convergences act in the net to significantly counter the effects of the synoptic-scale eddies in the vertically integrated angular momentum budget (though the planetary-scale heat fluxes also act as a positive feedback on the shift in the upper-tropospheric jets by amplifying the vertical wind shear). The negative feedback on the surface winds from planetary-scale topography is consistent with the well-known reduction in annular mode time scales associated with its imposition in simple models.

The spectrum of warmings generated by this set of experiments in principle provides an opportunity to test the applicability of fluctuation-dissipation theoremtype ideas to their tropospheric response. The results here are plausibly consistent with these ideas as described above, though a detailed analysis is beyond the scope of this work. Two points of caution, however, bear emphasizing: First, the correspondence between the stratospheric decorrelation time scales and the recovery time scale for sudden warmings is not robust; this has been noted for observations and comprehensive models by Hitchcock et al. (2013). Second, the tropospheric decorrelation time scales are relatively insensitive in these integrations to both the change in the time-mean latitude of the jet and to the extended persistence of the stratospheric anomalies produced by the weakened radiative damping rates. This is in fact consistent with the results of $\mathrm{CO}$ (who likewise saw little change in the tropospheric decorrelation time scales) but stands in contrast to sensitivity of the time scales to sudden warmings in a comprehensive model diagnosed by Simpson et al. (2011). Given the potential importance of the decorrelation time scales to the response of the tropospheric circulation to climate change (Kidston and Gerber 2010), a better understanding of the relationship between the decorrelation time scales and the composite picture presented here would seem desirable.

Acknowledgments. The authors acknowledge support from the Natural Sciences and Engineering Research Council and the Canadian Foundation for Climate and 
Atmospheric Sciences. We thank the Japan Society for the Promotion of Science for funding a summer visit to Kyoto University during which this collaboration began and the Centre for Global Change Science at the University of Toronto for supporting a subsequent visit. The linear wave model was provided by Nili Harnik. PH thanks Isla Simpson and Peter Haynes for helpful discussions.

\section{APPENDIX}

\section{Uncertainty Estimation}

\section{a. Time-averaged quantities}

Serial correlation is taken into account in the confidenceinterval estimation by reducing the degrees of freedom from the sample size $n$, following Zwiers and von Storch (1995). The number of effective degrees of freedom $n_{e}$ is computed from the autocorrelation function $\rho(t)$ of the given quantity using [their Eq. (4)]

$$
n_{e}=\frac{n}{1+2 \sum_{t=1}^{n-1}(1-t / n) \rho(t)} .
$$

The sum in the denominator was found to converge adequately if terms were retained up to the lag where the autocorrelation function first falls below $e^{-2}$.

\section{b. Annular mode time scales}

Annular mode time scales $\tau(p)$ were computed by performing a least squares fit of the computed autocorrelation functions at each pressure $p$ to the following form:

$$
\rho(t)=e^{-t / \tau} \cos (\alpha t)+\epsilon,
$$

using the first 50 days of lag. Including the cosine modulation was found to significantly improve the fit over a simple exponential. Note that according to the fluctuation dissipation theorem an autocorrelation function of this form would imply that the response to an external forcing be proportional to $\tau\left(\tau^{2}+\alpha^{2}\right)^{-1}$. The sensitivity of this quantity to the lower-stratospheric damping did not show any clearer a response than did the time scales $\tau$ themselves.

A rough estimate of the sampling error in these time scales was obtained by computing the autocorrelation function for each nonoverlapping 1000-day period of a run independently. The uncertainty in the sample mean was taken as a confidence interval for the time scale.

\section{REFERENCES}

Andrews, D. G., J. R. Holton, and C. B. Leovy, 1987: Middle Atmosphere Dynamics. Academic Press, 489 pp.

Baldwin, M. P., and T. J. Dunkerton, 2001: Stratospheric harbingers of anomalous weather regimes. Science, 294, 581-584, doi:10.1126/science.1063315.

, D. B. Stephenson, D. W. J. Thompson, T. J. Dunkerton, A. J. Charlton, and A. O'Neill, 2003: Stratospheric memory and skill of extended-range weather forecasts. Science, 301, 636640, doi:10.1126/science.1087143.

Charlton-Perez, A. J., and A. O'Neill, 2010: On the sensitivity of annular mode dynamics to stratospheric radiative timescales. J. Climate, 23, 476-484.

Cohen, J., and J. Jones, 2011: Tropospheric precursors and stratospheric warmings. J. Climate, 24, 6562-6572.

Dickinson, R. E., 1973: Method of parameterization for infrared cooling between altitudes of 30 and 70 kilometers. J. Geophys. Res., 78, 4451-4457.

Gerber, E. P., and L. M. Polvani, 2009: Stratosphere-troposphere coupling in a relatively simple AGCM: The importance of stratospheric variability. J. Climate, 22, 1920-1933.

, S. Voronin, and L. M. Polvani, 2008: Testing the annular mode autocorrelation time scale in simple atmospheric general circulation models. Mon. Wea. Rev., 136, 1523-1536.

—, and Coauthors, 2010: Stratosphere-troposphere coupling and annular mode variability in chemistry-climate models. J. Geophys. Res., 115, D00M06, doi:10.1029/2009JD013770.

Haigh, J. D., M. Blackburn, and R. Day, 2005: The response of tropospheric circulation to perturbations in lower-stratospheric temperature. J. Climate, 18, 3672-3685.

Harnik, N., 2009: Observed stratospheric downward reflection and its relation to upward pulses of wave activity. J. Geophys. Res., 114, D08120, doi:10.1029/2008JD010493.

— the vertical structure and variability of stratospheric planetary waves. J. Atmos. Sci., 58, 2872-2894.

Held, I. M., and M. J. Suarez, 1994: A proposal for the intercomparison of the dynamical cores of atmospheric general circulation models. Bull. Amer. Meteor. Soc., 75, 1825-1830.

Hitchcock, P., and T. G. Shepherd, 2013: Zonal mean dynamics of extended recoveries from stratospheric sudden warmings. J. Atmos. Sci., 70, 688-707.

,,-- and S. Yoden, 2010: On the approximation of local and linear radiative damping in the middle atmosphere. J. Atmos. Sci., 67, 2070-2085.

- _ - and G. L. Manney, 2013: Statistical characterization of Arctic polar-night jet oscillation events. J. Climate, 26, 20962116.

Holton, J. R., and C. Mass, 1976: Stratospheric vacillation cycles. J. Atmos. Sci., 33, 2218-2225.

Karoly, D. J., and B. J. Hoskins, 1982: Three dimensional propagation of planetary waves. J. Meteor. Soc. Japan, 60, 109-122.

Keeley, S. P. E., R. T. Sutton, and L. C. Shaffrey, 2009: Does the North Atlantic Oscillation show unusual persistence on intraseasonal timescales? Geophys. Res. Lett., 36, L22706, doi:10.1029/2009GL040367.

Kidston, J. and E. P. Gerber, 2010: Intermodel variability of the poleward shift of the austral jet stream in the CMIP3 integrations linked to biases in 20th century climatology. Geophys. Res. Lett., 37, L09708, doi:10.1029/2010GL042873.

Kohma, M., S. Nishizawa, and S. Yoden, 2010: Classification of polar-night jet oscillations and their relationship to fast and 
slow variations in a global mechanistic circulation model of the stratosphere and troposphere. J. Climate, 23, 6438-6444.

Kuroda, Y., and K. Kodera, 2001: Variability of the polar night jet in the Northern and Southern Hemispheres. J. Geophys. Res., 106 (D18), 20 703-20 713.

and — , 2004: Role of the Polar-night Jet Oscillation on the formation of the Arctic Oscillation in the Northern Hemisphere in winter. J. Geophys. Res., 109, D11112, doi:10.1029/ 2003JD004123.

Kushner, P. J., 2010: Annular modes of the troposphere and stratosphere. The Stratosphere: Dynamics, Transport, and Chemistry, Geophys. Monogr., Vol. 190, Amer. Geophys. Union, 59-91, doi:10.1029/2009GM000924.

_ , and L. M. Polvani, 2004: Stratosphere-troposphere coupling in a relatively simple AGCM: The role of eddies. J. Climate, 17, 629-639.

Laprise, R., and C. Girard, 1990: A spectral general circulation model using a piecewise-constant finite-element representation on a hybrid vertical coordinate system. J. Climate, 3, 32-52.

McLandress, C., and T. G. Shepherd, 2009: Impact of climate change on stratospheric sudden warmings as simulated by the Canadian Middle Atmosphere Model. J. Climate, 22, 5449-5463.

Mukougawa, H., T. Hirooka, and Y. Kuroda, 2009: Influence of stratospheric circulation on the predictability of the tropospheric Northern Annular Mode. Geophys. Res. Lett., 36, L08814, doi:10.1029/2008GL037127.

Perlwitz, J., and N. Harnik, 2004: Downward coupling between the stratosphere and troposphere: The relative roles of wave and zonal mean processes. J. Climate, 17, 4902-4909.

Plumb, R. A., and K. Semeniuk, 2003: Downward migration of extratropical zonal wind anomalies. J. Geophys. Res., 108, 4223, doi:10.1029/2002JD002773.
Polvani, L. M., and P. J. Kushner, 2002: Tropospheric response to stratospheric perturbations in a relatively simple general circulation model. Geophys. Res. Lett., 29, 1114, doi:10.1029/ 2001GL014284.

Scott, R. K., and L. M. Polvani, 2006: Internal variability of the winter stratosphere. Part I: Time-independent forcing. J. Atmos. Sci., 63, 2758-2776.

Simpson, I. R., M. Blackburn, and J. D. Haigh, 2009: The role of eddies in driving the tropospheric response to stratospheric heating perturbations. J. Atmos. Sci., 66, 1347-1365.

-, P. Hitchcock, T. G. Shepherd, and J. F. Scinocca, 2011: Stratospheric variability and tropospheric annular-mode timescales. Geophys. Res. Lett., 38, L20806, doi:10.1029/ 2011 GL049304.

Son, S.-W., and Coauthors, 2008: The impact of stratospheric ozone recovery on the Southern Hemisphere westerly jet. Science, 320, 1486-1489, doi:10.1126/science.1155939.

Song, Y., and W. A. Robinson, 2004: Dynamical mechanisms for stratospheric influences on the troposphere. J. Atmos. Sci., 61, 1711-1725.

Taguchi, M., T. Yamaga, and S. Yoden, 2001: Internal variability of the troposphere-stratosphere coupled system simulated in a simple global circulation model. J. Atmos. Sci., 58, 31843203.

Thompson, D. W. J., and S. Solomon, 2002: Interpretation of recent Southern Hemisphere climate change. Science, 296, 895-899, doi:10.1126/science.1069270.

Yoden, S., M. Taguchi, and Y. Naito, 2002: Numerical studies on time variations of the troposphere-stratosphere coupled system. J. Meteor. Soc. Japan, 80, 811-830.

Zwiers, F. W., and H. von Storch, 1995: Taking serial correlation into account in tests of the mean. J. Climate, 8, 336-351. 